\title{
Thromboprophylaxis in spinal surgery: A survey of current practice in South Africa
}

\author{
HW Jacobs MBChB(US) \\ Senior Registrar in Orthopaedic Surgery \\ I Zondagh MBChB(UP), FC(Orth)(SA) \\ Consultant in Spinal Surgery \\ PF Wessels MBChB(UP), MMed(Haem) \\ Consultant in Medical Oncology \\ University of Pretoria \\ Corresponding author: \\ Dr HW Jacobs \\ Steve Biko Academic Hospital \\ Tel: +27 123541000 \\ Email: hanswjacobs@gmail.com
}

\begin{abstract}
Background: Patients undergoing spinal surgery are at risk of developing venous thromboembolism (VTE) because of co-morbidities and immobilisation, but the morbidity of bleeding and haematoma formation complicates prophylaxis. A balance between VTE prevention and haematoma formation is therefore critical. Adding to the complexity is that there are currently no clear guidelines on managing these patients and little evidence in the literature. In order to improve management, it is imperative to first establish the current practice of thromboprophylaxis in spinal surgery in South Africa.

Methods: A survey of surgeons' peri-operative thromboprophylactic management was conducted with 112 spinal surgeons.

Results: The results indicated that a large group of surgeons did not follow a standardised protocol. Mechanical prophylaxis was not used optimally and more surgeons used chemical prophylaxis routinely than mechanical prophylaxis. The surgeons mostly agreed on the type of chemical prophylaxis used and the time of commencement thereof. Contrary to the literature, the surgeons in this study did not differentiate between the surgical approaches used.

Conclusion: There is currently no consensus on the management of thromboprophylaxis, as evident from the varying responses regarding treatment and complications. It is clear that mechanical prophylaxis is currently under-utilised. Spinal surgeons would benefit from a standardised protocol, and the vast majority of participants in the study agreed with this recommendation.
\end{abstract}

Key words: thromboprophylaxis, venous thromboembolism (VTE), spinal surgery, current practice, lowmolecular-weight heparin (LMWH)

http:/ / dx.doi.org/10.17159/2309-8309/2017/v16n1a8

\section{Introduction}

Thromboprophylaxis in spinal surgery is controversial. On the one hand, there is deep vein thrombosis (DVT) and pulmonary embolism (PE), which is potentially fatal, and on the other, the risk of bleeding and haematoma formation. Spinal epidural haematoma (SEH) has a high morbidity rate and includes possible neurological deficit. This can have deleterious consequences and emphasises the importance of a balance between venous thromboembolism (VTE) prevention and bleeding.
Venous thromboembolism is a medical complication but increases after any recent surgery. According to Virchow's triad the risk increases with spinal surgery owing to prolonged operative procedures, manipulation of great vessels (endothelial injury), prone positioning and postoperative bed rest (stasis). Patients are also often high-risk patients for DVT and their risk is compounded by increased age, smoking habit, hypertension, diabetes, cancer and dehydration (hypercoagulable state). 
The increased risk of VTE in spinal surgery has directed research towards safe and effective prophylactic regimens. Either mechanical or chemical prophylaxis or a combination can be used, depending on the surgeon's preference. Mechanical prophylaxis includes graduated compression stockings (GCS), intermittent pneumatic compressions (IPC), and inferior vena cava (IVC) filter placement. Apart from the IVC filter, which is not commonly used, mechanical prophylaxis is non-invasive with a low morbidity rate and can therefore be used routinely. ${ }^{1}$ Chemical prophylaxis, however, has a high morbidity rate and there is very little evidence in the literature regarding indications, use or benefits. Although the true incidence of thromboembolic events in spinal surgery remains unknown, the literature suggests that it ranges between 0.3 and $31.0 \% .^{2.3}$ This varies with surgical procedure, with the risk of DVT ranging from $1.0 \%$ in smaller spinal procedures (two-level laminectomies and smaller) up to $2.2 \%$ in major spinal surgeries (fusions and extensive laminectomies). Lumbar spinal surgery and anterior approach surgery also increase the risk of DVT and $\mathrm{PE} .{ }^{4}$ In contrast to this, bleeding and SEH formation in spinal surgery have severe consequences and can lead to neurological deficit. The incidence of $\mathrm{SEH}$, however, is much lower than that of DVT, ranging from 0.0 to $0.7 \%$, but never more than $1.0 \%$.

The increased risk of VTE in spinal trauma patients has been recognised. The use of chemical prophylaxis is usually indicated, but there is little consensus on thromboprophylaxis in elective spinal surgery. ${ }^{4}$ Although low-molecularweight heparin (LMWH) is regarded as safe in lumbar decompression surgery, it is advocated that no more than mechanical prophylaxis is needed in non-trauma spinal surgery. ${ }^{67}$

Recommendations and guidelines in the literature regarding thromboprophylaxis in spinal surgery are lacking and there is little consensus. It is left to the surgeon to weigh the risks and benefits of thromboprophylaxis for each patient. ${ }^{8,9}$ Much of the related research has been conducted by the North American Spine Society and little or no level one or level two evidence has been published regarding this, leaving the choice from a wide spectrum of management possibilities to the surgeon.

The objective of this study was to investigate the current practice of thromboprophylaxis in spinal surgery among surgeons in South Africa. A survey was conducted through a questionnaire to determine whether there is any consensus between spinal surgeons and their current treatment regimens. We also investigated whether a standardised protocol would benefit spinal surgeons in managing these patients and whether this survey could assist in the development of future preventative guidelines.

\section{Materials and methods}

The study has an observational cross-sectional design and was conducted through a survey administered via an anonymous questionnaire on the current practice of thromboprophylaxis in South Africa. It was directed specifically at elective thoracolumbar (TL) surgery. Ethical committee approval was obtained through our institution. The questionnaire was distributed to spinal surgeons who attended the South African Spine Society Congress in 2014 and their informed consent was obtained. Neurosurgeons and orthopaedic surgeons were included without differentiation. A total of 150 surgeons were invited to participate in the survey with 115 of the distributed questionnaires returned. Three of the 115 questionnaires collected were incomplete; therefore, 112 questionnaires were used in the study. The questionnaire covered the number of surgeries performed weekly (minor and major elective TL surgeries), the protocols used, differentiation between anterior and posterior approach surgeries, the methods of prophylaxis routinely used and complications seen, with both proximal and distal DVTs included as DVTs, in the respondents' practices.

The data obtained was analysed via cross-tabulation using the Fisher exact test and chi-squared test. Statistical significance was set at $p<0.5$. Cross-analysis was also performed between groups.

\section{Results}

Surgeons were grouped according to the number of surgeries they performed weekly. This could be an indication of their level of experience. The groups, Group A (0-3 surgeries a week), Group B (4-6 surgeries a week) and Group C ( $>6$ surgeries a week), are indicated in the chart, with most surgeons falling into Group A (Figure 1).

Of all of the surgeons surveyed, a large group of $25 \%$ did not follow any protocol regarding thromboprophylaxis and of the $75 \%$ that did, one-third based their protocol on personal experience. This indicates that up to $50 \%$ of the spinal surgeons did not apply evidence-based medicine in managing these patients. Cross-analysis showed that fewer surgeons in Group A applied a thromboprophylactic protocol than the average. This was statistically significant, with $p=0.012$.

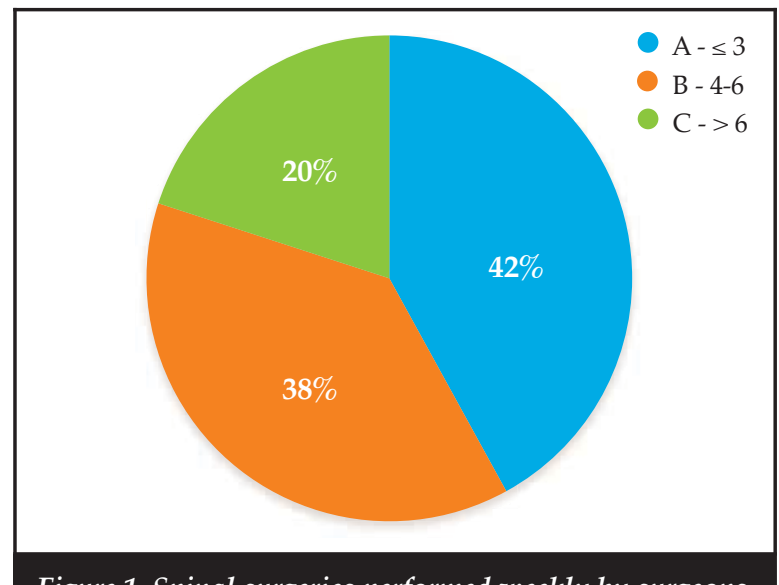




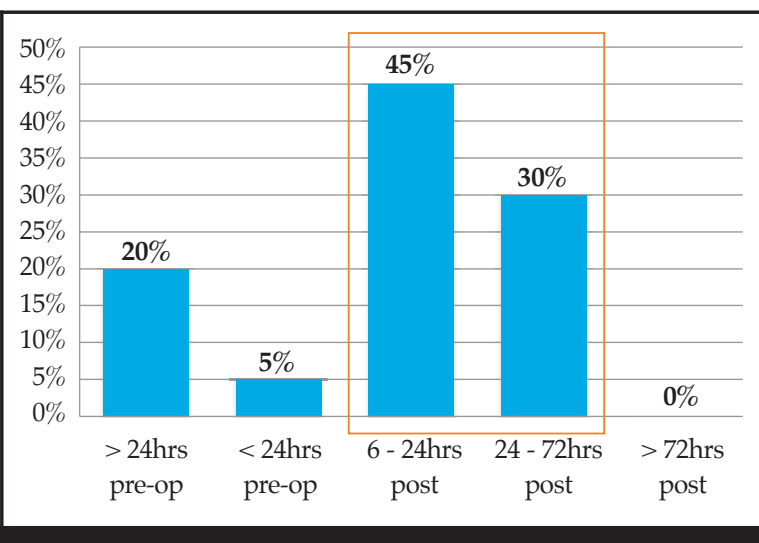

Figure 2. When chemical prophylaxisis is commenced

Mobility of patients is an important factor to consider with regard to prophylaxis, with greater consensus between surgeons in this regard, with $>50 \%$ continuing chemical prophylaxis until patient is mobile rather than a fixed time period of administration. There was also greater consensus regarding the time of commencement of chemical prophylaxis, with $75 \%$ of the surgeons starting 6-72 hours post-operatively (Figure 2).

In the survey, $93 \%$ of the surgeons used chemical prophylaxis routinely, compared with $85 \%$ who used any form of mechanical prophylaxis. For chemical prophylaxis, LMWH was most commonly used (Figure 3).

\section{Discussion}

The surgeons' perceived risk of DVT and PE was comparable to that reported in the literature because of the wide range of incidence. Of the surgeons, $83 \%$ indicated an incidence of between 0 and 5\% for DVT and 77\% indicated an incidence of $<2 \%$ for PE. The group that did not use chemical prophylaxis routinely perceived the risk to be lower (compared with the average) and the group that used chemical prophylaxis other than LMWH (unfractionated heparin, new oral anticoagulants and antiplatelets) perceived the risk to be higher (than the average) with $p=0.009$.

The surgical approach could influence the risk of the patient developing DVT. Anterior approach surgery is reported to have a higher risk of DVT and PE incidence because of the manipulation of great vessels. Therefore, some surgeons advise that thromboprophylactic management in the case of anterior approach surgery should be more aggressive.

Of the surgeons surveyed, $89 \%$ did not differentiate between anterior and posterior approach surgeries (Figure 4). This could be as a result of most of the surgeons already using chemical prophylaxis routinely regardless of the surgery performed. There was greater differentiation between anterior and posterior approach surgeries in Group $\mathrm{C}$ and this finding was statistically significant, with

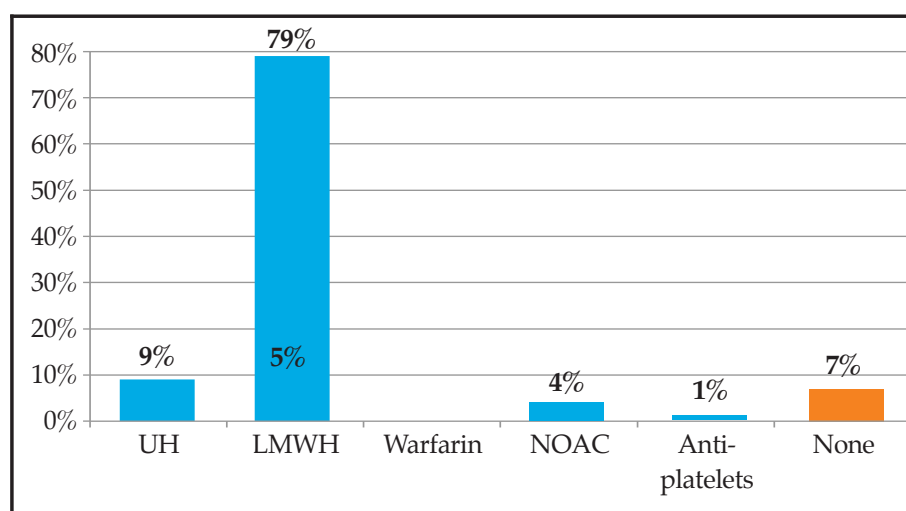

Figure 3. Routine use of chemical prophylaxis

$p<0.05$. It is possible that this might indicate their experience by being more aggressive with anterior approach surgery and also less prone to using chemical prophylaxis for all patients.

As mentioned, post-operative complications vary between DVT / PE and bleeding/haematoma formation. In the literature, it is reported that VTEs are more common than bleeding post-operatively, even though bleeding as a complication is difficult to define. When asked whether DVT/PE or bleeding/haematoma formation was more common in their practice as a complication, $69 \%$ of the surgeons responded that bleeding/haematoma formation was more common (Figure 5). This could be an indication either of their perception of any oozing of wounds as bleeding or of overuse of chemical prophylaxis in South Africa.

The literature varies with regard to the routine use of prophylaxis, with some researchers recommending that no more than mechanical prophylaxis is necessary in nontrauma spinal surgery. However, the literature strongly advocates the routine use of mechanical prophylaxis. Our results of the routine use of prophylaxis showed that more surgeons used chemical prophylaxis routinely than mechanical, which is concerning (Figure 6).

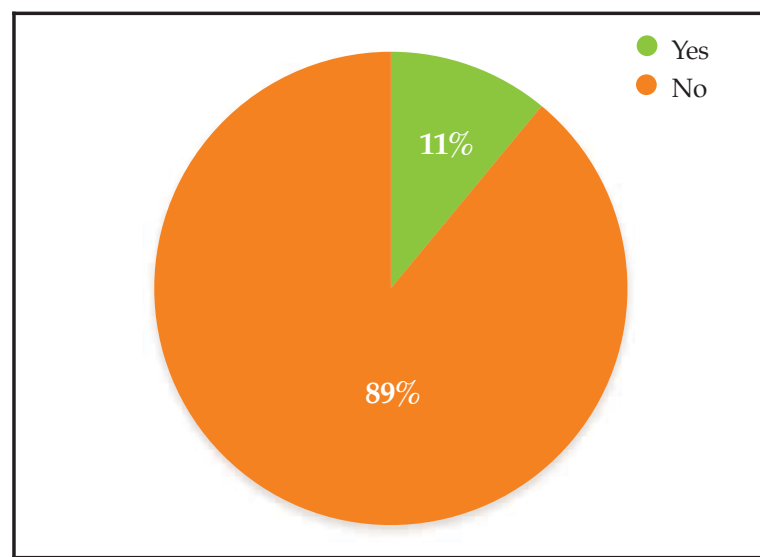

Figure 4. Does surgical approach influence prophylaxis? 


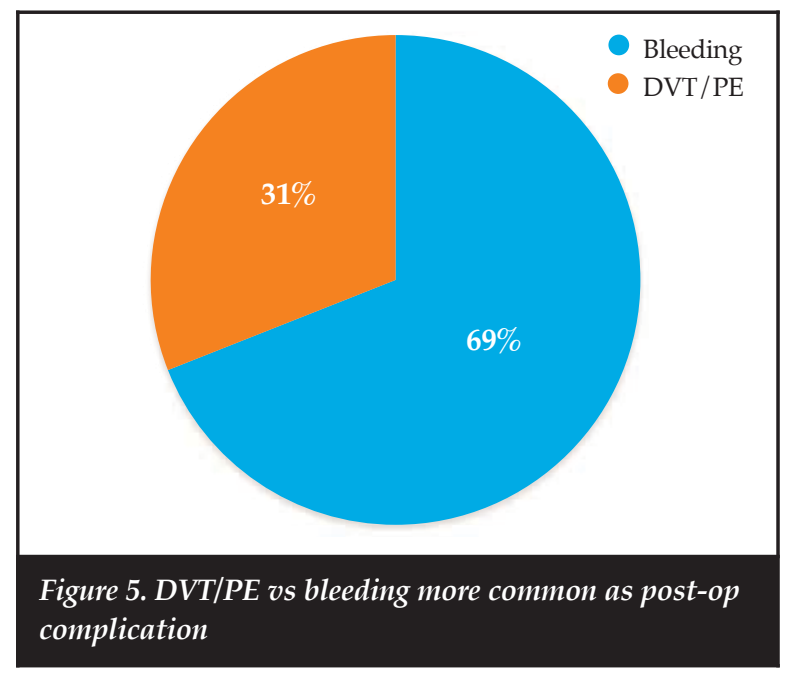

\section{Conclusion}

Guidelines on thromboprophylaxis in spinal surgery are clearly indicated, but the lack of evidence complicates this with insufficient published data to provide meaningful guidelines on the use of chemical prophylaxis.

Similar surveys have been conducted abroad, but this is the first in South Africa and is currently the largest survey on thromboprophylaxis in spinal surgery known internationally. Varying responses regarding treatment and complications, as well as the differences between the groups, indicate that there is currently no general consensus on the management of thromboprophylaxis in spinal surgery. From the literature, there is not much doubt about the benefits of applying mechanical thromboprophylaxis (ease of use and the low morbidity rate); however, the current practice shows it is used less routinely than chemical prophylaxis. It is therefore clear that mechanical prophylaxis is currently underused and that chemical prophylaxis is perhaps overused in South Africa.

Although this article does not present a protocol, it does stress the importance of this with $89 \%$ of participating surgeons agreeing that they would benefit from a published standardised protocol. This would be a major advancement in the management of spinal patients, especially with regard to chemical thromboprophylaxis. This survey will contribute to the possibility of future practice guidelines by knowledge of the current practice in South Africa but further research is necessary.

\section{Compliance with ethics guidelines}

I, HW Jacobs, hereby give written permission for the publication of my manuscript on 'Thromboprophylaxis in spinal surgery: A survey of current practice in South Africa' and that this article is the sole work of the co-authors and myself. This article has never been published and is submitted exclusively to the South African Orthopaedic Journal for publication.

Ethical committee approval was obtained through our institution.

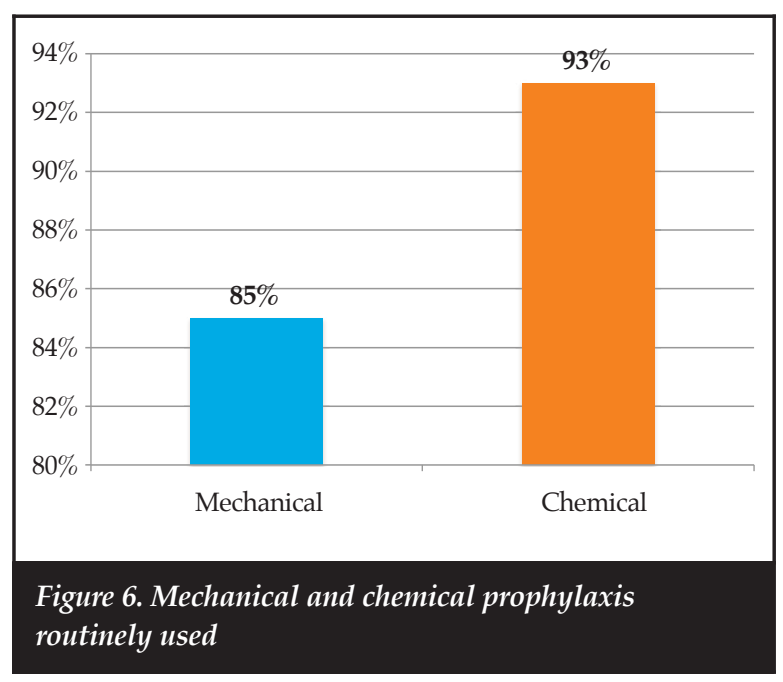

\section{References}

1. Kurtoglu M, Yanar H, Bilsel Y, Guloglu R, Kizilirmak S, Buyukkurt $\mathrm{D}$, et al. Venous thromboembolism prophylaxis after head and spinal trauma: intermittent pneumatic compression devices versus low molecular weight heparin. World J Surg. 2004 Aug;28(8):807-11.

2. Catre MG. Anticoagulation in spinal surgery. A critical review of the literature. Can J Surg. 1997 Dec;40(6):413-19.

3. Cunningham JE, Swamy G, Thomas KC. Does preoperative DVT chemoprophylaxis in spinal surgery affect the incidence of thromboembolic complications and spinal epidural hematomas? J Spinal Disord Tech. 2011 Jun;24(4):E31-4.

4. Audibert G, Faillot T, Vergnes MC, Bosson JL, Bernard C, Payen JF, et al. [Thromboprophylaxis in elective spinal surgery and spinal cord injury]. Ann Fr Anesth Reanim. 2005 Aug;24(8):928-34.

5. Glotzbecker MP, Bono CM, Wood KB, Harris MB. Postoperative spinal epidural hematoma: a systematic review. Spine 2010 May 1;35(10):E413-20.

6. Zhi-jian S, Yu Z, Giu-xing Q, Yi-peng W, Xi-sheng W, Hong $Z$, et al. Efficacy and safety of low molecular weight heparin prophylaxis for venous thromboembolism following lumbar decompression surgery. Chin Med Sci J. 2011 Dec;26(4):22126.

7. Ploumis A, Ponnappan RK, Sarbello J, Dvorak M, Fehlings MG, Baron E, et al. Thromboprophylaxis in traumatic and elective spinal surgery: analysis of questionnaire response and current practice of spine trauma surgeons. Spine 2010 Feb 1;35(3):323-29.

8. Bryson DJ, Uzoigwe CE, Braybrooke J. Thromboprophylaxis in spinal surgery: a survey. J Orthop Surg Res. 2012 Mar 29;7:14.

9. Al-Dujaili TM, Majer CN, Madhoun TE, Kassis SZ, Saleh AA. Deep venous thrombosis in spine surgery patients: incidence and hematoma formation. Int Surg. 2012 Apr-Jun;97(2):150-54.

10. North American Spine Society. Evidence-based clinical guidelines for multidisciplinary spine care: Antithrombotic therapies in spine surgery [Internet]. Burr Ridge: North American Spine Society; 2009 [cited 2016 Apr 23]. 96 p. Available from https:// www.spine.org/Documents/ ResearchClinicalCare/Guidelines / AntithromboticTherapies .pdf. 


\section{Further reading}

Barnes B, Alexander JT, Branch CL Jr. Postoperative Level 1 anticoagulation therapy and spinal surgery: practical guidelines for management. Neurosurg focus. 2004 Oct 15;17(4):E5.

Cheng JS, Arnold PM, Anderson PA, Fischer D, Dettori JR. Anticoagulation risk in spine surgery. Spine 2010 Apr 20;35(9 Suppl):S117-24.

Gerlach R, Raabe A, Beck J, Woszczyk A, Seifert V. Postoperative nadroparin administration for prophylaxis of thromboembolic events is not associated with an increased risk of hemorrhage after spinal surgery. Eur Spine J. 2004 Feb;13(1):9-13.

Janni W, Bergauer F, Rjosk D, Lohscheidt K, Hagena FW. [Prospective randomized study comparing the effectiveness and tolerance of various low-molecularweight heparins in high risk patients]. Zentralbl Chir. 2001 Jan;126(1):32-38.

Nicol M, Sun Y, Craig N, Wardlaw D. Incidence of thromboembolic complications in lumbar spinal surgery in 1,111 patients. Eur Spine J. 2009 Oct;18(10):1548-52.

Nourbakhsh A, Garges KJ. Lumbar synovial joint hematoma in a patient on anticoagulation treatment. Spine 2007 Apr 20;32(9):E300-302.

Raj D, Marshall RW. Prophylaxis against thromboembolism in spinal surgery. Arch Orthop Trauma Surg. 2008 Dec;128(12):1365-71.
Rocha E, Imberti D, Paschina E. Low-molecular-weight heparins: before or after surgery? New concepts and evidence: congress report from the Sigma Tau/ROVI Satellite Symposium (Rome, Italy, 13 November 2006). Clin Drug Investig. 2007;27(5):357-66.

Spanier DE, Stambough JL. Delayed postoperative epidural hematoma formation after heparinization in lumbar spinal surgery. J Spinal Disord. 2000 Feb;13(1):46-49.

Steib A, Hadjiat F, Skibba W, Steib JP, French Spine Surgery Society. Focus on perioperative management of anticoagulants and antiplatelet agents in spine surgery. Orthop Traumatol Surg Res. 2011 Oct;97(6 Suppl):S102-6.

Yoshioka K, Kitajima I, Kabata T, Tani M, Kawahara N, Murakami $\mathrm{H}$, et al. Venous thromboembolism after spine surgery: changes of the fibrin monomer complex and D-dimer level during the perioperative period. J Neurosurg Spine 2010 Nov;13(5):594-99.

This article is also available online on the SAOA website (www.saoa.org.za) and the SciELO website (www.scielo.org.za). Follow the directions on the Contents page of this journal to access it. 